\title{
Semantic generalization as a function of associative value of stimuli
}

\author{
SPURGEON COLE AND ROBERT L. WILLIAMS ${ }^{t}$ \\ WEST GEORGIA COLLEGE
}

The study attempted to determine if the smooth gradient usually obtained in primary generalization could be obtained for semantic generalization. Six generalization words were ranked in terms of associative strength to the conditioned stimuli. It was predicted that the greater the associative strength between CSs and each generalization stimulus, the greater the generalization. Shock was used to condition the S's Galvanic Skin Response to the CSs. The magnitude of semantic generalization was then assessed by measuring the GSR to the generalization stimuli. Although the generalization gradient followed a decelerating curve, the GSR amplitude was not completely consistent with the measured associative strength of the stimulus words.

The generalization of conditioned responses is an empirically demonstrated phenomenon. Numerous experiments have shown that if an organism has been conditioned to respond to a particular stimulus, another stimulus similar to the original will also elicit the response. In many of the demonstrations of generalization, gradients have appeared with the magnitude of the generalized response varying inversely with the distance of $S_{2}-{ }_{n}$ from $S_{1}$ along a given physical dimension. Experiments dealing with primary generalization have found that as the amount of similarity between the stimuli decreases, the tendency for the related stimulus to elicit the response also decreases. The gradient that is obtained is relatively smooth and regular.

The present experiment attempted to determine if the same smooth and regular gradient can be obtained in semantic generalization. In semantic generalization the dimension along which generalization occurs is not a function of the physical attributes of the stimulus, but of similarity in meaning (Razran, 1935-36). For example, if a conditioned GSR is established to the stimulus word "style," the response will also occur to the synonym "fashion" (Razran, 1939).

It appears logical that if the degree of "relatedness" between the words used as CS and the ones used as generalization stimuli could be accurately measured, a generalization gradient similar to the gradient in primary generalization would be obtained. The "relatedness," if measured on an equal interval level, would be similar to jnds obtained for physical stimuli. The greater the degree of relatedness in meaning between the conditioned words and generalization words, the greater the generalization.

Bousfield, Whitmarsh, \& Danich (1958) have devised a method of measuring the "relatedness" or strength of association between two stimuli by determining the number of common responses elicited by the two stimuli. In the present experiment, the words "anger" and "rage" were chosen as conditioned stimuli and the words "temper," "hate," "mad," "fight," "fear," and "red" were chosen as generalization stimuli. The initial selection of these words was based on the number of times the generalization stimuli were given as associations to the words "rage" and "anger" on a simple word association test. The associative strength or degree of relatedness of the generalization stimuli was then determined by the Bousfield et al (1958) method. The predicted generalization for the words used as generalization stimuli were in the following order: "temper," "hate," "mad," "fear," "fight," and "red." If this method accurately measures relatedness or similarity in meaning between the generalization words and the conditioned words, it should be possible to predict greater generalization from the conditioned stimulus to "temper" than to "hate" and more to "hate" than to "mad," etc.

The theoretical generalization gradient based on the above measure of relatedness was compared to the actual gradient by conditioning $S$ to give GSR responses to the words "rage" and "anger" and then obtaining GSR responses to the generalization words.

\section{Subjects and Apparatus}

A single group of 15 male Ss were employed in the experiment. Their ages ranged from 18 to 26 and all were naive for this experiment. The apparatus for the experiment included an electrostatically shielded room, an armchair facing a viewing screen on which words could be flashed, a tachistoscope, a shock unit, and GSR instrumentation.

\section{Procedure}

The experimental procedure involved four steps: (1) determination of pain threshold; (2) adaptation trials; (3) training trials; (4) generalization trials.

(1) Pain threshold. Beginning with an intensity of 15 milliwatts and a duration of $100 \mathrm{msec}$., the shock level was systematically increased by 10 milliwatt steps until the $S$ reported that the stimulus was painful.

Immediately after the S's pain threshold was established, the following instructions were read:

In the rectangle of light in front of you a single word will be flashed at intervals. It will appear so briefly that you will not always be sure what the word is. Every time a word is flashed on, I want you to depress the telegraph key with your left hand and then tell me what you think each word 


\section{FIGURE I. \\ GENERALIZATION GRADIENT BASED \\ ON GSR RESPONSES}

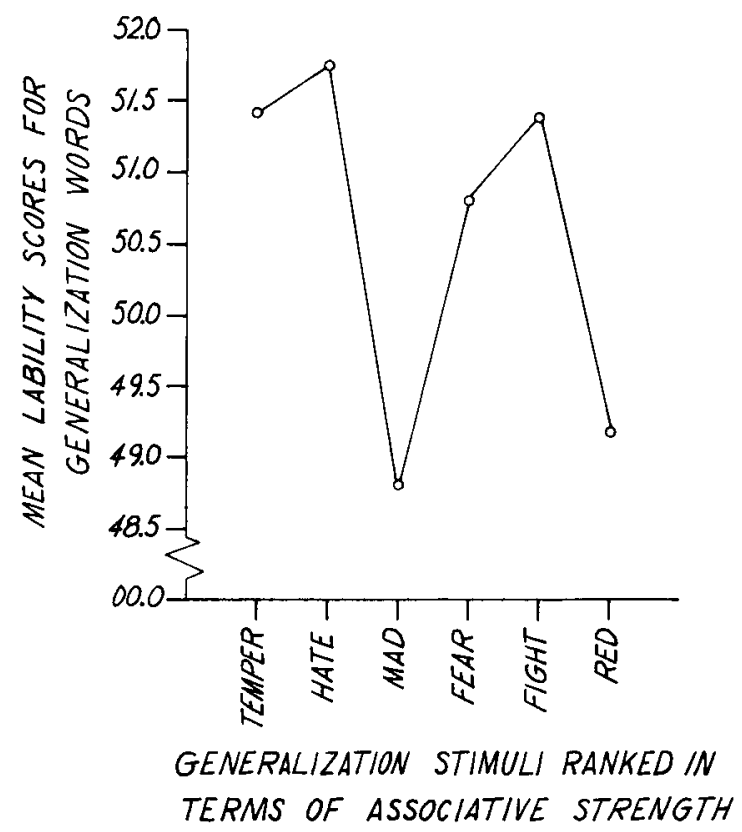

Fig. 1. Generalization gradient based on GSR response.

is, even if you have to make a wild guess.

(2) Adaptation trials. The $S$ was presented a list of 30 words. These words were not part of the experimental list and served only to adapt the $S$ to the experimental situation and to determine the threshold level at which the $S$ could recognize the words. Each stimulus word was tachistoscopically presented at intervals varying randomly between 31 and $39 \mathrm{sec}$.

(3) Training trials. The training trials followed with no interruption and without further instruction. The training trials consisted of 12 presentations of each CS along with 24 presentations of neutral words. The two CSs ("rage" and "anger") were followed by shock on all 24 occurrences. The generalization stimuli were not included in the training trials.

(4) Generalization trials. The list of words presented during the generalization trials consisted of six neutral words and the six generalization words. Each generalization stimulus was presented three times.

\section{Results}

Inspection of the generalization data (Fig.1) indicates that the amplitude of GSR response followed only a rough gradient which varied with degree of association of the test stimuli to the CSs. Although the gradient followed a decelerating curve, the GSR amplitude was not directly related to the associative strength of all stimulus words. Although the stimulus word "mad" was ranked high in associative value to the CSs, there was less generalization to it than to the least associated word. Responses to the other stimulus words were generally consistent with the theoretical gradient, but two others showed some discrepancy between associative strength and amplitude of generalized GSR.

Conditioning was apparent as the amplitude of GSR was found to vary significantly $(p<.01)$ with word connotation. Response amplitude was significantly higher for the generalized stimuli than for neutral words. Discussion

The Bousfield method of obtaining associative strength of semanticly related generalization words appears at least partially inadequate. Although clear evidence of generalization was obtained, the present study failed to find a smooth gradient for semantic generalization based on the Bousfield et al (1958) method of measuring associative strength of generalization stimuli. As previously noted, there was practically no generalization to the stimulus word "mad," although the word was supposedly strongly related to the condition stimuli. However, if this particular stimulus is removed from the gradient, the other stimuli follow a rough, but obviously decelerating curve.

The lack of agreement between the predicted and actual gradients may not be due entirely to the inadequacy of the Bousfield method. The length of the word may also play some part in determining the amount of GSR response to the stimulus word. In the present study, the length of the word could have influenced generalization in at least two ways. The shorter word would be more easily recognized, thus, reducing the amount of fear of the situation. Secondly, since the condition stimuli were four and five letters long, primary generalization to the longer words would have been enhanced and reduced to all three letter words. These factors may well have influenced the form of the generalization gradient.

\section{References}

Bousfield, W. A., Whitmarsh, G. A., \& Danick, J. J. Partial response identity in verbal generalization. Psychol. Rep., 1958, 4, 703-713.

Razran, G. R. S. Conditioned responses: An experimental study and a theoretical analysis. Arch. Psychol., 1935-1936, 191. (573)

Razran, G. H. S. A quantitative study of meaning by a conditioned salivary technique. Science, 1939, 90, 89-90.

\section{Note}

1. The authors would like to express appreciation to Herbert Zimmer (University of Georgia) for his contributions to this paper. 